\title{
An observational feasibility study to assess the safety and effectiveness of intranasal fentanyl for radiofrequency ablations of the lumbar facet joints
}

\author{
This article was published in the following Dove Press journal: \\ Journal of Pain Research \\ 10 February 2017 \\ Number of times this article has been viewed
}

\author{
Michael W Bartoszek' \\ Amy McCoart ${ }^{2}$ \\ Kyung-soo Jason Hong ${ }^{3}$ \\ Chelsey Haley ${ }^{2}$ \\ Krista Beth Highland ${ }^{4}$ \\ Anthony R Plunkett ${ }^{1}$ \\ 'Department of Anesthesiology, \\ Womack Army Medical Center, Fort \\ Bragg, NC, ${ }^{2}$ Clinical Investigations, \\ Defense and Veterans Center for \\ Integrative Pain Management, Henry \\ M. Jackson Foundation, Womack \\ Army Medical Center, Fort Bragg, \\ NC, ${ }^{3}$ Research Department, The \\ Center for Clinical Research, \\ Sceptor Pain Foundation, Winston \\ Salem, NC, ${ }^{4}$ Defense and Veterans \\ Center for Integrative Pain \\ Management, Henry M. Jackson \\ Foundation, Uniformed Services \\ University, Bethesda, MD, USA
}

\begin{abstract}
Purpose: The purpose of the present observational, feasibility study is to assess the preliminary safety and effectiveness of intranasal fentanyl for lumbar facet radiofrequency ablation procedures.

Patients and methods: This cohort observational study included 23 adult patients. Systolic and diastolic blood pressures, heart rate, oxygen saturation percent, Pasero Opioid-Induced Sedation Scale score, and the Defense and Veterans Pain Rating Scale pain score were assessed prior to the procedure and intranasal fentanyl $(100 \mu \mathrm{g})$ administration and every 15 minutes after administration, up to 60 minutes post administration. Follow-up of patient satisfaction with pain control and treatment was assessed 24 hours after discharge. The primary outcome was safety as evidenced by adverse events. Secondary outcomes included the above-mentioned vital signs and pain ratings.
\end{abstract}

Results: No adverse events occurred in the present study and all participants maintained an acceptable level of awareness throughout the assessment period. One-way repeated measures analyses of covariance tests with Bonferroni-adjusted means indicated that oxygen saturation, blood pressure, and heart rate changed from baseline, whereas pain scores were lower at postadministration levels compared with baseline. Finally, the majority of participants reported being satisfied with pain control and treatment.

Conclusion: Preliminary evidence indicates that intranasal fentanyl is safe and effective for lumbar facet radiofrequency ablation procedures. Future rigorous randomized control trials are needed to confirm the present results and to examine the effects of intranasal fentanyl on intraoperative and postoperative opioid use.

Keywords: analgesia, spinal procedures, chronic pain, sedation

\section{Introduction}

The field of interventional pain management is challenged to provide effective and safe care while mitigating treatment-related risks. Sedation is used to enhance patient comfort, with approximately half providers offering sedation during lumbar spine and cervical spine procedures. ${ }^{1}$ However, deeper sedation is associated with an elevated risk of catastrophic neurological complications. ${ }^{2}$ Such risk may be especially salient for patients receiving procedures involving spinal nerves. For example, traumatic spinal cord injury was more commonly reported in claims among patients who were unresponsive, received sedation, or received general anesthesia during cervical procedures for chronic pain. ${ }^{3}$ Combined with guidelines set forth by the American Society
Correspondence: Amy McCoart Department of Clinical Investigations, Womack Army Medical Center, 2817 Reilly Road, Building 2517, Fort Bragg, NC 28310 , USA

$\mathrm{Tel}+19109076691$

Fax + I 9109077870

Email Amy.m.mccoart.ctr@mail.mil 
of Anesthesiologists (ASA) ${ }^{4}$ and the International Sedation Task Force, ${ }^{5}$ patient wakefulness during procedures involving spinal nerves is vital and efforts to minimize the risk of deeper sedation while providing adequate pain management are imperative.

Intravenous fentanyl is used by pain clinics as a sole agent or combined with a benzodiazepine to minimize pain during procedures. Previous studies have found that intravenous fentanyl up to $250 \mu \mathrm{g}$ reliably produces adequate relaxation in about 8 minutes without resulting in deep sedation. ${ }^{6,7}$ Although effective, patients receiving intravenous fentanyl require significant time and resource-consuming efforts from medical providers. To address the burden of intravenous fentanyl use, intranasal fentanyl was developed. Trials in animal models revealed that intranasal fentanyl provided an instantaneous analgesic effect due to the immediate distribution into the bloodstream in rats. ${ }^{8}$ In humans, pectin-formulated intranasal fentanyl allows adherence to the nasal cavity without waste, has an $89 \%$ bioavailability and mean $\mathrm{T}_{\text {max }}$ of 12 minutes after a $100-\mu \mathrm{g}$ single-dose administration, and provides a mean action duration of 58 minutes. ${ }^{9-11}$ However, less is known whether the intranasal route provides a favorable safety profile in minimizing the risk of heavy sedation and providing adequate pain relief during spinal procedures for chronic pain.

Intranasal fentanyl has demonstrated safety and effectiveness in both pediatric and adult populations, across a number of conditions. Intranasal fentanyl provided similar levels of behavioral distress and reduced pain levels after abscess incision and drainage procedures of children compared with those receiving intravenous morphine. ${ }^{12}$ Similarly, a Cochrane review of pediatric trials indicated that intranasal fentanyl is likely effective for acute moderate-to-severe pain. ${ }^{13}$ In addition to evidence indicating efficacy and tolerability of intranasal fentanyl in children, additional research has cited similar effects in adults.

For adults, intranasal fentanyl is approved by the Food and Drug Administration for the management of breakthrough cancer pain in patients aged $\geq 18$ years who are tolerant to opioid therapy. In a double-blind, placebo-controlled clinical study examining intranasal fentanyl for breakthrough cancer pain in participants receiving opioid therapy for back pain, sum of pain intensity difference scores at 30-minute post-intranasal fentanyl administration reflected greater breakthrough cancer pain relief compared with participants receiving a placebo. ${ }^{3}$ After 3 months of self-administering intranasal fentanyl for breakthrough cancer pain, 34 patients in an observational, prospective, cohort study reported a mean dose of 87-119 $\mu \mathrm{g}$, as well as improved sleep and good-toexcellent medication effectiveness. ${ }^{14}$

In addition to breakthrough cancer pain, other trials have examined the effectiveness of intranasal fentanyl for other procedures inducing acute pain. In a randomized doubleblind, double-dummy, two-way, crossover study comparing intravenous and intranasal fentanyl routes for pain during third-molar extraction, patients reported statistically similar analgesic effects and nasally related adverse events, but those receiving intranasal fentanyl were less likely to use rescue medication. Previously, $50 \mu \mathrm{g}$ intranasal fentanyl was effective in reducing pain intensity in the hour following drain removal in a sample of healthy women receiving breast augmentation or reduction. ${ }^{15}$ Finally, in a case study examining breakthrough pain in patients with vertebral compression fracture due to multiple myeloma, patients responded better to the pectin gel intranasal fentanyl treatment when compared with previous opioids treatment and reported a higher personal satisfaction. ${ }^{16}$

There is some preliminary evidence that higher doses of intranasal fentanyl may carry greater risk of adverse events. In a pilot study examining patient-controlled intranasal fentanyl during childbirth, patients used an average of $734 \mu \mathrm{g}$ over 3.6 hours and reported satisfactory to excellent pain relief. However, the risk of neonatal respiratory depression increased with higher fentanyl doses. Additional evidence also demonstrates the efficacy and safety of intranasal fentanyl in pediatric patients. ${ }^{17}$ Taken together, a single intranasal fentanyl dose (eg, $100 \mu \mathrm{g}$ ) may be a safe option for reducing pain, while minimizing the risk of sedation.

The purpose of the present observational, feasibility study is to assess the preliminary safety and effectiveness of intranasal fentanyl (Lazanda) ${ }^{9}$ for lumbar facet radiofrequency ablation procedures. Intranasal fentanyl has not been closely studied in procedures involving spinal nerves. Radiofrequency ablation of the medial branches of the posterior primary ramus is a commonly performed pain procedure lasting $\sim 30$ minutes and well suited for preliminary testing of this pectin-based intranasal fentanyl. In examining our primary outcome, we hypothesize that intranasal fentanyl will be safe and well tolerated, as evidenced by a lack of adverse events. In examining our secondary outcomes, we hypothesize that participants receiving intranasal fentanyl will remain easily aroused or awake and alert through the time of discharge, without significant elevations in vital signs, and report reduced pain after intranasal fentanyl administration, as well as high treatment satisfaction. 


\section{Materials and methods}

This cohort observational study was registered with clinicaltrials.gov (NCT02571634) and approved by the Womack Army Medical Center Institutional Review Board. Male and female patients were recruited through referral by a Womack Army Hospital Interdisciplinary Pain Management Center staff member. Both opioid naïve and experienced participants were included. Inclusion and exclusion criteria are listed in Table 1.

After patients provided written informed consent, final screening and initial assessment were completed by collecting vital signs (systolic blood pressure [SBP], diastolic blood pressure [DBP], heart rate [HR], and oxygen saturation percent $\left[\mathrm{O}_{2}\right]$ ), Pasero Opioid-Induced Sedation Scale (POSS) score, and the Defense and Veterans Pain Rating Scale (DVPRS) pain score. ${ }^{18-20}$ Applicable health history, ASA classification, medications, allergies to medications, demographic information, and procedure type (unilateral or bilateral) were also collected. Intranasal fentanyl (100 $\mu \mathrm{g})$ was administered immediately prior to entering the procedure room by one of the three providers involved in the study, $\sim 10$ minutes prior to procedure start. Intranasal fentanyl comes in an 800- $\mu \mathrm{g}$ metered sprays in $100 \mu \mathrm{g}$ individual doses. The remaining $700 \mu \mathrm{g}$ was wasted and witnessed into a charcoal pouch (Figure 1). Lumbar facet radiofrequency ablation procedures were conducted consistent with standard of care at the study institution and included local anesthetic (lidocaine) at the needle insertion sites (Figure 2).

Vital signs, POSS scores, and DVPRS pain scores were obtained at 15, 30, 45, and 60 minutes post-administration.

Table I Inclusion and exclusion criteria

\begin{tabular}{ll}
\hline Inclusion criteria & Exclusion criteria \\
\hline Military beneficiary & Known allergy to fentanyl, \\
Age I8+ years & naloxone, or meperidine \\
ASA health status classification & Allergic rhinitis with active \\
rating $\leq$ III & rhinorrhea \\
Scheduled for a radiofrequency & Pregnant or breastfeeding \\
nerve ablation of lumbar facet & Asthma or chronic obstructive \\
joints (unilateral or bilateral) & pulmonary disease requiring \\
& frequent rescue medications \\
& Hepatic insufficiency with abnormal \\
& liver enzymes \\
& Noted decreased mental function \\
& Heart rate $<50$ bpm \\
& Blood pressure $<90$ mmHG systolic \\
& or <60 mmHG diastolic \\
& Myasthenia gravis \\
& Acute narrow angle glaucoma \\
& Current medication with a known \\
dangerous interaction with fentanyl
\end{tabular}

Abbreviation: ASA, American Society of Anesthesiologists

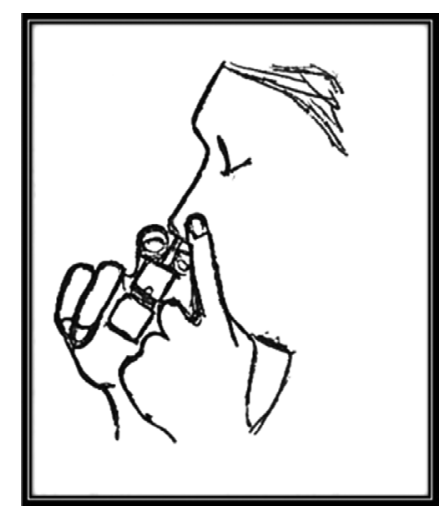

Figure I Illustration of the intranasal administration procedure.

Notes: The patient presses down on the release mechanism until an audible click indicates the full dose has been released. Patients are instructed to breathe-in gently through their nose during medication release and then breathe-out through their mouth once after spraying. Image courtesy of $\mathrm{KJ}$ Hong.

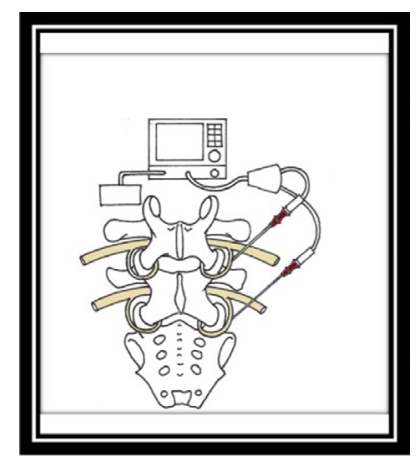

Figure 2 Illustration of radiofrequency ablation of the lumbar facet joints. Notes: A special (radiofrequency) needle is inserted alongside the medial or lateral branch nerves using X-ray guidance (fluoroscopy). A small electrical current in the needle ensures that it is in the target location and at a safe distance from other nerves. Image courtesy of KJ Hong.

In the event, the participant was discharged prior to the 60-minute assessment, a final assessment occurred at discharge. Finally, study staff called participants 24 hours post-procedure to assess satisfaction with both pain control during the procedure and their overall treatment. Responses were rated on a 5-point Likert scale with responses ranging from "very unsatisfied" to "very satisfied". ${ }^{21}$

The primary outcome for this study was to assess the safety of intranasal fentanyl in acute procedures as evidenced by adverse events. As additional safety parameters, secondary outcomes also assessed patient sedation (POSS score) and vital signs (SBP, DBP, HR, and oxygen saturation) in the 60 minutes following intranasal fentanyl administration. Finally, additional secondary outcomes included reported changes in pain from the initial DVPRS score. Univariate statistics examined means (standard deviation [SD]) and frequencies of study variables. To test the primary hypothesis, frequencies of adverse events were reported. To test the secondary hypotheses related to sedation (POSS) and satisfac- 
tion (patient satisfaction scales), frequencies were reported. Additional secondary hypotheses regarding DVPRS, oxygen saturation, SBP, DBP, and HR were tested using one-way repeated measures analysis of covariance (rmANCOVA). An rmANCOVA holds several advantages, including the ability to model covariates, thereby minimizing variance accounted for by extraneous and confounding variables. Such models also produce adjusted marginal means, which can then be used to more accurately estimate sample sizes for future clinical trials. Furthermore, rmANCOVA pairwise comparisons were assessed using the Bonferroni-adjusted significance tests, which reduced the likelihood of type I errors by conservatively estimating differences while accounting for multiple tests and alpha inflation. Age, body mass index (BMI), ASA classification, procedure length, and procedure type were included as covariates. According to hypotheses, Bonferroni-adjusted significance tests examined differences between baseline and post-administration values. All analyses were completed using SPSS Statistics 24.0 (IBM Corporation, Armonk, NY, USA).

\section{Results}

Participants $(\mathrm{N}=23)$ were mostly male $(\mathrm{n}=22)$ and White $(n=19)$, as well as African American $(n=2)$ and others $(n=2)$, with an average age of $40.5 \pm 7.7$ years and BMI of $29.77 \pm 2.39$ $\mathrm{kg} / \mathrm{m}^{2}$. Participants ASA classifications were either I $(\mathrm{n}=6)$ or II $(n=17)$. Most participants $(n=19)$ were not taking opioids within the 4 weeks prior to the procedure. Most had a unilateral radiofrequency ablation procedure $(n=14)$. The average radiofrequency ablation procedure time for single (32.36 $\pm 7.86 \mathrm{~min})$ and bilateral $(38.78 \pm 14.10 \mathrm{~min})$ procedures were not significantly different $(t=1.25, p=0.24)$. In addition, all participants retained oxygen saturation levels at $95 \%$ or greater at each interval, aside from one participant who had a saturation of $94 \%$ at the 45 -minute interval, but returned to $97 \%$ at the 60 -minute interval.

Consistent with the primary hypothesis, all participants tolerated the procedure without complications, as there were no adverse events. Similarly, our secondary hypotheses regarding safety were also supported, such that all participants had a POSS score corresponding to "alert and awake" at every time point, aside from one participant who had a POSS score corresponding to "slightly drowsy, easily aroused" at 15-, 30-, and 45-minute intervals prior to returning to "alert and awake" by discharge.

Three rmANCOVAs examined longitudinal changes in oxygen saturation, SBP, DBP, and HR. Oxygen saturation $\left(F[4,64]=0.62, p=0.65\right.$, partial $\left.\eta^{2}=0.04\right)$, SBP $(F[4$, $64]=2.41, p=0.06$, partial $\left.\eta^{2}=0.13\right)$, DBP $(F[4,64]=1.10$, $p=0.37$, partial $\left.\eta^{2}=0.06\right)$, and $\operatorname{HR}(F[4,64]=1.10, p=0.37$, partial $\eta^{2}=0.06$ ) did not significantly change overall across time. Consistent with the hypotheses, post-hoc Bonferroniadjusted comparison tests indicated that oxygen saturation, SBP, DBP, and HR did not significantly vary between baseline and post-administration intervals. Adjusted means (SD) are shown in Table 2.

Results from an rmANCOVA indicated that DVPRS scores did not significantly change overall across the study period $\left(\mathrm{F}[4,68]=0.85, p=0.50\right.$, partial $\left.\eta^{2}=0.05\right)$. Post hoc Bonferroni-adjusted comparison tests indicated that DVPRS scores were lower at the 15 -minute $(p=0.06), 30$-minute $(p=0.02), 45$-minute $(p=0.004)$, and 60-minute $(p<0.001)$ intervals. Therefore, the hypothesis that pain would be less at post-administration than at baseline was supported. Adjusted means (standard error) are shown in Table 2.

Finally, the hypothesis that participants would endorse satisfaction with pain control during the procedure and overall treatment at 24 hours post-procedure was supported. The majority of participants reported being very satisfied (57\%) and satisfied (30\%) with pain control, with the remaining participants reporting being neither satisfied nor unsatisfied (13\%). Similarly, majority of participants also reported being very satisfied (57\%) and satisfied (30\%) with their overall treatment experience, with the remaining participants reporting being neither satisfied nor unsatisfied (13\%). No participants reported being somewhat unsatisfied or very unsatisfied on either measure.

Table 2 Adjusted means (SE) of DVPRS and vital signs at baseline and post-administration intervals

\begin{tabular}{llllll}
\hline Outcomes & Mean (SE) & & & \\
\cline { 2 - 6 } & Baseline & I5 minutes & $\mathbf{3 0}$ minutes & 45 minutes & 60 minutes \\
\hline Oxygen saturation, \% & $97.73(0.23)$ & $98.36(0.25)$ & $98.00(0.34)$ & $97.18(0.28)$ & $97.27(0.25)$ \\
Systolic blood pressure, mmHg & $123.36(2.42)$ & $124.09(2.12)$ & $122.64(2.86)$ & $125.00(2.22)$ & $119.68(1.62)$ \\
Diastolic blood pressure, $\mathrm{mmHg}$ & $80.23(2.22)$ & $79.50(2.69)$ & $81.23(2.31)$ & $82.86(1.67)$ & $79.82(2.01)$ \\
Heart rate, bpm & $72.32(2.44)$ & $68.05(2.56)$ & $69.18(2.37)$ & $68.64(2.12)$ & $68.59(2.11)$ \\
DVPRS & $4.70(0.31)$ & $3.61(0.42)^{\mathrm{a}}$ & $2.52(0.50)^{\mathrm{b}}$ & $2.52(0.43)^{\mathrm{b}}$ & $1.65(0.35)^{\mathrm{b}}$ \\
\hline
\end{tabular}

Note: Displayed means and SEs are adjusted for American Society of Anesthesiologists classification, age, body mass index, procedure length, and procedure type. Superscript alphabets denote significant differences between baseline value and post-administration interval: ${ }^{a} p<0.10,{ }^{b} p<0.05$.

Abbreviations: bpm, beats per minute; DVPRS, Defense and Veterans Pain Rating Scale; SE, standard error. 


\section{Discussion}

This non-randomized cohort study demonstrated the safety and preliminary effectiveness of intranasal fentanyl for use in lumbar facet radiofrequency ablation procedures. Reducing sedation risk is key to preventing adverse events and procedural complications. ${ }^{1,2}$ Patients tolerated the medication well with no recorded adverse events. Vital signs remained stable and patients remained awake and alert during the procedure and reported reduced pain after intranasal fentanyl administration. Patients also reported satisfaction with pain control and overall treatment on the day following their procedure. Overall, the present findings support future, larger explorations of intranasal fentanyl during outpatient procedures, especially those involving spinal nerves.

In the present study, a single intranasal fentanyl dose was set at $100 \mu \mathrm{g}$. In a previous pilot study, there was a reported elevated risk of neonatal respiratory depression when mothers utilized patient-controlled intranasal spray at much higher doses (eg, $734 \mu \mathrm{g}$ and greater over a 3.6-hour period) than those used in the present study. ${ }^{14}$ Increasing fentanyl dose is associated with increased analgesia; however, doubling the dose does not double the action duration..$^{10}$ In weighing the risks and benefits, $100 \mu \mathrm{g}$ may provide optimal pain relief and minimal risk and future studies are needed to examine whether variations in doses or administration frequency would alter the balance between risks and benefits.

Intranasal fentanyl administration may not only mitigate risk of heavy sedation but also reduce challenges associated with intravenous access. In particular, intranasal fentanyl may be a desirable choice for acute medical procedures pertinent to military service members (eg, battlefield triage), allowing providers to quickly move patients unencumbered by intravenous lines in emergency situations. In two prior observational studies examining the safety and efficacy of intranasal fentanyl in the pre-hospital (ambulance) setting, there were no serious adverse effects and patients reported analgesia effectiveness. ${ }^{22}$ In its current packaging, intranasal fentanyl may lead to medication waste. However, intranasal administration may reduce the need for personal use of additional materials typically required for intravenous administration, and medication administration time. Thus, intranasal fentanyl may be more favorable than other administration routes due to its effectiveness, safety, and cost-effectiveness.

The present trial was not designed to compare intranasal fentanyl with a placebo or active controls such as intravenous fentanyl and oral benzodiazepines. Therefore, we are unable to definitively state that the reductions in pain were due to a local anesthetic or intranasal fentanyl. Self-reported pain is a subjective measure, thus we included additional objective outcomes (eg, blood pressure, HR) to examine effectiveness, as well as safety. Future empirical endeavors of intranasal fentanyl may benefit from additional measures of pain such as time to rescue analgesia.

The majority of participants in the present study were not using opioids in the month prior to the procedure and we did not include a formal assessment of substance-related disorders. However, patients with active, diagnosed substance-related disorders are first referred to treatment and the procedure is then performed when remission occurs. Given the small number of participants who were using opioids in the month prior to surgery $(n=4)$, we are not able to make conclusions regarding efficacy specifically for individuals receiving long-term opioid treatment or those with substance-related disorders. Given the opioid crisis in the USA, it will be important to examine the effects of intranasal fentanyl for acute pain in patients receiving long-term opioid medications and monitor opioid use in the weeks and months that follow the procedure.

In addition, local anesthetic was administered per standard of care at our institution and we did not examine the degree to which anesthetics dose and volume impact study outcomes. Future clinical trials would benefit from either standardizing anesthetic dose or including it as an additional moderator of treatment outcomes.

\section{Acknowledgments}

This study was made possible by the grant and study medication provided by Depomed. We would also like to thank the Pain Clinic staff at Womack for their assistance and support of this study.

\section{Disclosure}

The authors report no conflicts of interest in this work, in addition; the views expressed herein are those of the authors and do not reflect the official policy of the Department of the Army, Department of Defense, or the U.S. Government.

\section{References}

1. Kim N, Delport E, Cucuzzella T, Marley J, Pruitt C. Is sedation indicated before spinal injections? Spine (Phila Pa 1976). 2007;32(25): E748-E752.

2. Neal J, Bernards C, Hadzic A, et al. ASRA practice advisory on neurologic complications in regional anesthesia and pain medicine. Reg Anesth Pain Med. 2008;33(5):404-415.

3. Rathmell JP, Michna E, Fitzgibbon DR, Stephens LS, Posner KL, Domino KB. Injury and liability associated with cervical procedures for chronic pain. Anesthesiology. 2011;114(4):918-926.

4. Bhananker SM, Posner KL, Cheney FW, Caplan RA, Lee LA, Domino KB. Injury and liability associated with monitored anesthesia care Anesthesiology. 2006;104(2):228-234.

5. Mason K, Green S, Piacevoli Q. Adverse event reporting tool to standardize the reporting and tracking of adverse events during procedural sedation: a consensus document from the World SIVA International Sedation Task Force. Br J Anaesth. 2011;108(1):13-20. 
6. Manchikanti L, Pampati V, Damron KS, et al. A randomized, prospective, double-blind, placebo-controlled evaluation of the effect of sedation on combined diagnostic cervical and lumbar facet joint nerve blocks. Pain Physician. 2004;7:301-309.

7. Manchikanti L. Evaluation of Lumbar facet joint nerve blocks in managing chronic low back pain: a randomized, double-blind, controlled trial with a 2-year follow-up. Int J Med Sci. 2010;7(3):124-135.

8. Hoekman JD, Ho RJ. Enhanced analgesic responses after preferential delivery of morphine and fentanyl to the olfactory epithelium in rats. Anesth Analg. 2011;113(3):641-651.

9. Lazanda fentanyl nasal spray for breakthrough pain now available. Oncology Times. 2011;33(21):15.

10. Kaasa S, Moksnes K, Nolte T, Lefebvre-Kuntz D, Popper L, Kress HG. Pharmacokinetics of intranasal fentanyl spray in patients with cancer and breakthrough pain. J Opioid Manag. 2010;6(1):17-26.

11. Foster D, Upton R, Christrup L, Popper L. Pharmacokinetics and pharmacodynamics of intranasal versus intravenous fentanyl in patients with pain after oral surgery. Ann Pharmacother. 2008;42(10):1380-1387.

12. Fenster D, Dayan P, Babineau J, Aponte-Patel L, Tsze D. Randomized trial of intranasal fentanyl versus intravenous morphine for abscess incision and drainage. Pediatr Emerg Care. Epub 2016 Jul 12.

13. Murphy A, O’Sullivan R, Wakai A, et al. Intranasal fentanyl for the management of acute pain in children. Cochrane Database Syst Rev. 2014;10:CD009942.

14. Thronæs M, Popper L, Eeg M, Jaatun E, Kvitberg M, Kaasa S. Efficacy and tolerability of intranasal fentanyl spray in cancer patients with breakthrough pain. Clin Ther. 2015;37(3):585-596.

15. Veldhorst-Janssen NM, Fiddelers AA, van der Kuy PH, et al. Pharmacokinetics, analgesic effect, and tolerability of a single preprocedural dose of intranasal fentanyl in patients undergoing drain removal after breast reduction or augmentation surgery: a prospective, randomized, doubleblind, placebo-controlled study. Clin Ther. 2010;32(7):1427-1436.
16. Bujedo BM, Karlsson B. Fentanyl in a pectin gel treating breakthrough pain in vertebral compression fracture due to multiple myeloma: a descriptive study of three cases. J Opioid Manag. 2016;12(3):197-203.

17. Kerr D, Taylor D, Evans B. Patient-controlled intranasal fentanyl analgesia: a pilot study to assess practicality and tolerability during childbirth. Int J Obstet Anesth. 2015;24(2):117-123.

18. Nisbet A, Mooney-Cotter F. Comparison of selected sedation scales for reporting opioid-induced sedation assessment. Pain Manag Nurs. 2009;10(3):154-164.

19. Buckenmaier C 3rd, Galloway KT, Polomano RC, McDuffie M, Kwon N, Gallagher RM. Preliminary validation of the defense and veterans pain rating scale (DVPRS) in a military population. Pain Med. 2013;14(1):110-123.

20. Polomano RC, Galloway KT, Kent ML, et al. Psychometric testing of the defense and veterans pain rating scale (DVPRS): a new pain scale for military population. Pain Med. 2016;17(8):1505-1519.

21. Matell M, Jacoby J. Is there an optimal number of alternatives for Likert scale items? study I: reliability and validity. Edu Psychol Meas. 1971;31(3):657-674.

22. Johnston S, Wilkes GJ, Thompson JA, Ziman M, Brightwell R. Inhaled methoxyflurane and intranasal fentanyl for prehospital management of visceral pain in an Australian ambulance service. Emerg Med J. 2011;28(1):57-63.

23. Rx List. Flunisolide nasal solution (Flunisolide nasal spray $0.025 \%$ ) drug information: medication guide and patient information - prescribing information at Rx List Web site. Available from: http://www.rxlist. com/flunisolide-nasal-solution-drug/medication-guide.htm. Accessed November 7, 2016.

24. Doctree.in. Radio Ablation of Nerves Facet Rhizolysis (Cervical/ Thoracic/Lumbar) Web site. Available from: https://doctree.in/knowledgebranch/radiofrequency-ablation-nerves-facet-rhizolysis/. Accessed November 7, 2016.
Journal of Pain Research

\section{Publish your work in this journal}

The Journal of Pain Research is an international, peer reviewed, open access, online journal that welcomes laboratory and clinical findings in the fields of pain research and the prevention and management of pain. Original research, reviews, symposium reports, hypothesis formation and commentaries are all considered for publication.

\section{Dovepress}

The manuscript management system is completely online and includes a very quick and fair peer-review system, which is all easy to use. Visit http://www.dovepress.com/testimonials.php to read real quotes from published authors. 